Boise State University

ScholarWorks

Psychological Sciences Faculty Publications and

Presentations

Department of Psychological Science

2-1-2011

\title{
Concurrent Herb-Prescription Medication Use and Health Care Provider Disclosure Among University Students
}

Cindy E. McCrea

Boise State University

Mary E. Pritchard

Boise State University

\section{(c) $(1) \Theta \Theta$}

This is an author-produced, peer-reviewed version of this article. (C) 2009, Elsevier. Licensed under the Creative Commons AttributionNonCommercial-NoDerivatives 4.0 International License (https://creativecommons.org/licenses/by-nc-nd/4.0/). The final, definitive version of this document can be found online at Complementary Therapies in Medicine, doi: 10.1016/j.ctim.2010.12.005 
NOTICE: This is the author's version of a work accepted for publication by Elsevier. Changes resulting from the publishing process, including peer review, editing, corrections, structural formatting and other quality control mechanisms, may not be reflected in this document. Changes may have been made to this work since it was submitted for publication. The definitive version has been published in Complementary Therapies in Medicine, Volume 19, Issue 1, 2011. DOI: 10.1016/j.ctim.2010.12.005

\title{
Concurrent Herb-Prescription Medication use and Health Care Provider Disclosure among University Students
}

\author{
Cindy E. McCrea \\ Boise State University \\ Mary E. Pritchard \\ Boise State University
}

\begin{abstract}
Objectives. To determine the extent to which college students are intermixing mood-altering herbs with prescription medications and whether they are disclosing this information to their health care providers.

Design and Setting. A nonrandom sample was drawn from the student body of a Northwestern state university $(\mathrm{n}=305)$.

Methods. In November 2008 participants completed an online survey detailing herb use, disclosure to health care providers and herb/medicinal intermixing.

Results. There were no demographic differences between herb users and non-users. Most herb usage was self-prescribed $(60 \%)$ and undisclosed to healthcare providers (only $25 \%$ of herb users disclosed to a healthcare provider). $34 \%$ of herb users used them to treat a mood disorder. Of herb users, thirteen percent had simultaneously used herbs and prescription medication in the last year. In addition, herb users who intermixed herbs with prescription medications had higher depression and anxiety scores than those who did not intermix.

Conclusions. College student herb use is primarily self prescribed and undisclosed to healthcare professionals, who may prescribe pharmaceuticals that interact negatively with herbals. Physician awareness and query is invaluable for the prevention of adverse herb and drug interactions.
\end{abstract}

Natural products, such as herbal remedies, rank in the top four treatments used to combat anxiety and top three for depression. ${ }^{1}$ In fact, anxiety and depression are in the top three most common ailments treated with complementary and alternative medicine (CAM) ${ }^{2}$ Adverse effects of these herbal treatments are usually rare, and mostly occur in cases where individuals have consumed high doses over prolonged time periods. ${ }^{3}$ However, little is known about serious consequences that might be induced by interactions between these herbs and psychotropic medication or other prescribed medications. ${ }^{4}$

Herbs with psychotropic implications, such as St. John's wort, chamomile, valerian root and kava kava are of particular interest as their use is often aimed at psychological symptoms. The effectiveness and safety of St. John's wort (SJW) for the treatment of depression has undergone more rigorous research than many other commonly used herbal remedies. Research indicates that although SJW has a very safe profile when used alone, it has a high potential for adverse drug interaction. ${ }^{5}$ Mixture of SJW with MAOI or SSRI antidepressants, for example, can result in a serious health condition known as serotonin syndrome. ${ }^{6,7}$ Serotonin syndrome occurs when the body is flooded with excess serotonin and can lead to muscle twitching, restlessness, breakdown of skeletal musculature, kidney failure and ultimately death. ${ }^{8}$ Additionally, in as little as four to ten days SJW can cause some medications to metabolize too quickly thus lowering plasma concentrations and rendering treatment less effective or ineffectual. ${ }^{5}$ Medications that SJW is known to adversely affect in this way include oral contraceptives, ${ }^{9}$ antiretroviral medications used in the treatment of AIDS such as Nevirapine and Indinivar, ${ }^{10,11}$ Digoxin, used to treat congestive heart failure, ${ }^{12}$ and fexofenidine or Allegra, used to treat allergies. ${ }^{13}$

Likewise, other herbal remedies used to treat mood disorders are thought to change the effects of prescription medications or exacerbate symptoms in dangerous ways. For example, valerian root and lemon balm may enhance the effects of other central nervous system depressants. ${ }^{6,14}$ Lemon balm may interact with thyroid medication and/or thyroid disease. ${ }^{14}$ Kava kava may enhance the effects of alcohol and benzodiazepines. ${ }^{6}$ Passion flower and skullcap have so little research regarding possible drug interactions that none can be identified with certainty. However given their sedative effects, they may enhance the effects of other central nervous system depressants. ${ }^{3,14}$ 
NOTICE: This is the author's version of a work accepted for publication by Elsevier. Changes resulting from the publishing process, including peer review, editing, corrections, structural formatting and other quality control mechanisms, may not be reflected in this document. Changes may have been made to this work since it was submitted for publication. The definitive version has been published in Complementary Therapies in Medicine, Volume 19, Issue 1, 2011. DOI: 10.1016/j.ctim.2010.12.005

\section{Herbal remedy and prescription medication intermixing}

A 1998 study estimated that $18.4 \%$ of all U. S. prescription users--or 15 million adults - had concurrently used prescription medication and herbal remedies and/or high dose vitamins in the last 12 months. ${ }^{15}$ The few more recent studies considering concurrent use have verified that a significant portion of the population may simultaneously use herbs and drugs; ${ }^{16,17}$ however, this phenomenon has not been well researched in college student populations.

\section{Herbal remedy use disclosure}

Research indicates that CAM users do not report alternative treatment use to their traditional health care providers, who may prescribe pharmaceuticals. ${ }^{1,17,18}$ In a review of twelve published studies, researchers found that nondisclosure of CAM use to traditional health care providers was occurred approximately $43 \%$ of the time. ${ }^{18}$ Interestingly, CAM users are much more likely to discuss their biomedical treatment details, such as prescription medications, to their alternative treatment provider. ${ }^{19,20}$

Although this trend of more complete disclosure to alternative therapists creates an avenue for the interception of drugs and herbs that may interact, few CAM users have their treatment supervised by an alternative treatment provider. ${ }^{2}$ This nominal use of CAM practitioners suggests that CAM users are self-prescribing treatment. Considering that herbal remedy users are usually not supervised by an alternative practitioner, ${ }^{2}$ disclosure to a traditional health care provider remains crucial for the prevention of possibly dangerous drug and herb intermixing.

\section{College students and herbal remedy usage}

Herbal use in college populations is reportedly much larger than that of the general population. ${ }^{27-29}$ A 2004 study reported overall herb use in a college student population at $52 \%(\mathrm{n}=1754)$ and, similar to general population studies, friends, family and self were the major modes of information and advice about herbals. ${ }^{27}$ The reported goal of herbal remedy use for $14.8 \%$ of users was emotional stability ${ }^{27}$ reinforcing the role that herbals play in mood management. Surprisingly, a high percentage of students who reported taking prescription and over-the-counter medications also reported using herbs: over $70 \%$ of students who were taking medications for depression were also using herbals. ${ }^{27}$ These findings indicating prevalence of herb use and the psychological motivations of that use in college student populations have been replicated. ${ }^{27-29}$ However, much less is known about disclosure of CAM use to health care providers or concurrent use of herbs and pharmaceuticals in this population.

\section{Present Study}

The purpose of this study is to ascertain: 1) what percentage of a college student population is using herbal remedies with psychological implications, 2) whether that use has been prescribed by or disclosed to a health care provider, and 3) is that herb use concurrent with pharmaceutical use?

\section{Methods}

A non random sample $(n=305)$ of college students at a Northwestern state university provided responses to an online survey regarding the use of herbs and pharmaceuticals. All respondents were enrolled in an introductory psychology course. This survey was one of many opportunities for students to fulfill research credits. The survey was administered through Qualtrics.com, an online survey program, and took anywhere from 15-25 minutes to complete. Institutional Review Board approval for this study was obtained and informed consent was implied with completion of the anonymous survey.

\section{Instrument}

All participants were asked a series of questions relating to a) demographics, b) use of herbs in the past 12 months, c) prescription drug use in the past 12 months, and d) to complete anxiety and depression inventories.

Participants who reported use of herbs in the past 12 months were further asked about details regarding that herb use. First, type of herb used was collected. Respondents were asked to choose any herbs they had used within the past year from a list of herbs used to alter mood. The list included St. John's wort, kava kava, lemon balm, skull cap, 
NOTICE: This is the author's version of a work accepted for publication by Elsevier. Changes resulting from the publishing process, including peer review, editing, corrections, structural formatting and other quality control mechanisms, may not be reflected in this document. Changes may have been made to this work since it was submitted for publication. The definitive version has been published in Complementary Therapies in Medicine, Volume 19, Issue 1, 2011. DOI: 10.1016/j.ctim.2010.12.005

passion flower, chamomile and valerian root. They were also allowed to select that they had used other not listed herbs within the past year. Herb users were also asked to identify who prescribed the herbs: self, medical personal, alternative health care practitioner, or other. By selecting "yes", "no" or "I don't/didn't have this type of a health care provider to inform," herb users indicated both having had and informed a general health care, alternative and mental health practitioner of their herb use. Finally, herb users noted the reason for their herb use: to help with sleep, to help with anxiety, to help with depression, or other.

Respondents were then asked whether they had taken any prescription medicine in the past year. If a respondent indicated the use of prescription medications in the past year, he/she was asked to list the type of prescription taken. Depression was measured using the Center for Epidemiological Studies Depression Scale (CES-D) which has been widely used and validated. ${ }^{30}$ Anxiety was measured using the Clinical Anxiety Scale (CAS), also widely accepted as a valid and robust measure. ${ }^{31}$

\section{Results}

In those who responded, age ranged from 18 to 50 years $(M=20.72, S D=4.87)$. The mode age was $18(\mathrm{n}=102)$. Forty-two percent were male $(\mathrm{n}=123)$ and $58 \%$ were female $(\mathrm{n}=168)$. Approximately $90 \%$ of participants were Caucasian. Twenty-five percent $(\mathrm{n}=77)$ of respondents reported herb usage in the past year.

\section{Herb Users and Non Herb Users Differences}

The mean age of herb users $(20.55$ years, sd $=4.78)$ was not significantly different that of non herb users $(21.09$, sd $=5.04), \mathrm{t}(300)=-.87$. The average response score on the CAS for all participants was $20.99(\mathrm{sd}=15.54)$, herb users' score $(22.8, \mathrm{sd}=14.32)$ was not different from that of non-herb users $(20.18, \mathrm{sd}=13.14), \mathrm{t}(292)=-1.53$. Similarly, the mean CES-D depression score $(14.77, \mathrm{sd}=10.44)$ was not significantly different for herb users $(16.17, \mathrm{sd}=11.7)$ and non herb users $(14.17, \mathrm{sd}=9.82), \mathrm{t}(292)=1.51$. Other key characteristics of herb users vs. nonherb users can be seen in Table 1 .

\section{Mode of Prescription and Disclosure}

Only $18 \%$ of herbal use was prescribed by a medical doctor (11\%) or alternative treatment provider (7\%); the remainder was self-prescribed (60\%) and/or prescribed by "other, e.g., health store employee, advice of a friend" $(40 \%)$. Of the sixty respondents who reported having a general health care provider and using an herbal remedy, only $25 \%$ disclosed their herbal usage to their general health care provider. Similarly, of the 38 respondents who reported using herbs in the last twelve months and seeing a counselor/therapist, only $18 \%$ disclosed their herbal use to that mental health care provider. Only $12 \%$ of herb users who had an alternative health care provider reported disclosing that usage to them.

\section{Intermixing of Herbs and Prescription Drugs}

Thirteen percent $(\mathrm{n}=39)$ of all respondents answered "yes" when asked if they had simultaneously used an herb and prescription medication in the last twelve months. The most common herbs to be used by individuals who reported concurrent use of pharmaceuticals and herbs are reported in Figure 1. Significant effects for both depression and anxiety scores on herb-drug intermixing were found: $\mathrm{t}(285)=4.12, \mathrm{p}<.001 ; \mathrm{t}(286)=4.69, \mathrm{p}<.001$. Individuals who intermixed herbs with prescription medicines had higher anxiety $(\mathrm{M}=28.85, \mathrm{SD}=15.57)$ and depression scores $(\mathrm{M}=21.67, \mathrm{SD}=12.36)$ than those who did not intermix herbs and prescription medicines (Anx: $\mathrm{M}=19.54$, $\mathrm{SD}=12.71$; Dep: $\mathrm{M}=13.53, \mathrm{SD}=9.67$ ) As displayed in Figure 2, when considering past 12 month herb use by past 12 month drug use, herb use was highest in respondents whole also took sedatives (48\%) and antidepressant (42\%).

\section{Comment}

This exploratory research was aimed at better understanding college students' use of herbs that are commonly utilized to alter mood. The mode of prescription, disclosure to health care professionals and rate of intermixing with prescription medication were explored. 
NOTICE: This is the author's version of a work accepted for publication by Elsevier. Changes resulting from the publishing process, including peer review, editing, corrections, structural formatting and other quality control mechanisms, may not be reflected in this document. Changes may have been made to this work since it was submitted for publication. The definitive version has been published in Complementary Therapies in Medicine, Volume 19, Issue 1, 2011. DOI: 10.1016/j.ctim.2010.12.005

\section{Prescription and Disclosure}

This study found that, similar to previous reports, ${ }^{2}$ most herb use was unsupervised by healthcare professionals: of the $25 \%$ of the sample that did use herbals in the past twelve months, only $11 \%$ were prescribed by a medical doctor while $7 \%$ were prescribed by an alternative provider. The remainder was self or otherwise prescribed. This is also consistent with earlier reports that self, friends and family are most often the sole source of information about herb use. $^{2,25}$

Alone, the finding that herbs use is mostly self-prescribed is unsettling, but the low disclosure rates that this research confirms provide an even greater cause for concern. Only $25 \%$ of herb users who had a general health care provider disclosed their herb use to that provider. Similarly, only 18 and 12 percent of herb users who had a counselor/therapist or alternative treatment provider, respectively, reported that herb use to them. This low disclosure rate, consistent with existing research, ${ }^{16}$ sets the stage for drug and herb intermixing with possibly dangerous consequences.

\section{Mixture of Herbs and Prescription Drugs}

The mixture of herbs and prescription medicines has been evaluated in the general population. National estimates around $18 \%$ for drug and herb intermixing were calculated in a 1998 study ${ }^{15}$ and more recently a 2005 study found that $38 \%$ of its respondents were taking both prescription and natural products. ${ }^{17}$ This study reported rates lower than both population studies, finding that $13 \%$ of all respondents mixed the use of herbs and prescription medication in the last 12 months. Of note is the possible inclusion of products other than herbs in the 2005 study's grouping: natural products. Similarly, the 1998 study included high dose vitamins. More research is needed to understand these discrepancies.

Of specific interest, $20 \%$ of all SJW users were also using a prescription drug. Thirty-three percent of SJW users were also taking medication which is known to have negative implications when mixed with SJW (oral contraceptives and allergy medication). No SJW users were found to also be taking antidepressants (MAOIs or SSRIs) which can precipitate serotonin syndrome. The herbs that were most commonly mixed with prescription medication were valerian root, "other" and chamomile. The most common medications mixed with herbs were oral contraceptives, allergy medication and pain medication. The first two have been shown to interact adversely with SJW. ${ }^{9,13}$

What is most alarming is the fact that herb users who did intermix with prescriptions reported higher depression and anxiety scores. It is not known whether those higher scores might be the result of herb-prescription intermixing or whether intermixing is the result of higher scores. Regardless, this is alarming and makes it even more important that herb users with mood symptomology alert their health care providers about their herb usage. Congruently, health care professionals who treat populations exhibiting high mood disorder symptomology should be aware of possible intermixing and question their patients about herb use.

\section{Limitations}

Limitations of this study include a small herb user sample size, and the scope of the questions asked. Three hundred and five students completed the questionnaire but only 77 respondents had used herbs in the last 12 months. In addition, many specifics about non herb users, such as the use of health care professionals, were not collected.

\section{Conclusion}

Despite these limitations, the present study indicates that non-disclosure of herbal usage to health care providers is prevalent in college students. Given the potential harmful effects from intermixing certain herbs with certain pharmaceuticals, health care providers (including campus health and wellness officials) need to be aware that patients/clients may be using herbal supplements that may influence mood or interact with prescription medicine. This may be especially true if the patient is a college student as herb use rates in this population are higher than that of the general population. Thus, healthcare providers may wish to directly ask about herbal supplement usage before prescribing medication. This is particularly true of mental health care professionals as the trend captured in earlier 
NOTICE: This is the author's version of a work accepted for publication by Elsevier. Changes resulting from the publishing process, including peer review, editing, corrections, structural formatting and other quality control mechanisms, may not be reflected in this document. Changes may have been made to this work since it was submitted for publication. The definitive version has been published in Complementary Therapies in Medicine, Volume 19, Issue 1, 2011. DOI: 10.1016/j.ctim.2010.12.005

literature is furthered here; individuals are self prescribing herbs to alter mood. Health care providers may wish to explain the potential side effects of combining prescription medicines with herbal supplements when they write prescriptions. Campus health and wellness centers may wish to offer workshops on the potential hazards of intermixing herbs and prescription medications.

Future studies should examine the reasons for and specific herb use among college populations. Also, a more detailed survey might shed light on which herbs and medications are most likely to be mixed. A closer look into student attributes (traditional vs. non- traditional) may shed light on the variance in published herbal use rates and, further, predict use. 
NOTICE: This is the author's version of a work accepted for publication by Elsevier. Changes resulting from the publishing process, including peer review, editing, corrections, structural formatting and other quality control mechanisms, may not be reflected in this document. Changes may have been made to this work since it was submitted for publication. The definitive version has been published in Complementary Therapies in Medicine, Volume 19, Issue 1, 2011. DOI: 10.1016/j.ctim.2010.12.005

\section{References}

1. Barraco D, Valencia G, Riba AL, Nareddy S, Draus CB, Schwartz SM. Complementary and alternative medicine (CAM) use patterns and disclosure to physicians in acute coronary syndromes in patients. Complement Ther Med. 2005; 13:34-40.

2. Barnes PM, Powell-Grinder E, McFann K, Nahin RL. Complementary and alternative medicine use among adults: United States, 2002. Semin Integr Med, 2004;2:54-71.

3. Wong AC, Smith M, Boon HS. Herbal remedies in psychiatric practice. General Psychiatry. 1998;55:1033-1044.

4. Chan E. Quality of efficacy research in complementary and alternative medicine. JAMA. 2008;299:2685-2686.

5. Izzo AA. Drug interactions with St. John's Wort (Hypericum perforatum): a review of the clinical evidence. Int J Clin Pharmacol Ther. 2004;42:139-148.

6. Beaubrun G, Gray GE. A review of herbal medicines for psychiatric disorders. Psychiatr Serv (Chic). 2000;51:1130-1134.

7. Hamerness P, Basch E, Ulbright C, et al. St. John's Wort: A systematic review of adverse effects and drug interactions for the consultation psychiatrist. Psychosomatics. 2003;44:271-282.

8. Yee A, Wijdicks E. A perfect storm in the emergency department. Neurocritical Care. April 2010;12(2):258260.15. Hall SD, Wang Z, Huang SM, et al. The interaction between St. John's wort and an oral contraceptive. Clin Pharmacol Ther 2003;74:525-35.

9. Hall SD, Wang Z, Huang SM, et al. The interaction between St. John's wort and an oral contraceptive. Clin Pharmacol Ther 2003;74:525-35.

10. de Maat M, Hoetelmans R, Mathôt R, et al. Drug interaction between St John's wort and nevirapine. AIDS. 2001;15:420-421.

11. Piscitelli SC, Burstein AH, Chaitt D, Alfaro RM, Falloon J. Indinavir concentrations and St John's wort. Lancet, 2000;355:547 -548.

12. Dasgupta, A. Herbal Supplements and Therapeutic Drug Monitoring: Focus on Digoxin Immunoassays and Interactions With St. John's Wort. Ther Drug Monit, 2008; 30(2):212-217.

13. Wang Z, Hamman MA, Huang SM, Lesko LJ, Hall SD. Effect of St John's wort on the pharmacokinetics of fexofenadine. Clin Pharmacol Ther. 2002;71:414-420.

14. Holcomb SS. Common herb drug interactions: What you should know. The Nurse Practitioner, 2009;34(5):2126.

15. Eisenburg DM, Davis, RB, Ettner SL et al. Trends in Alternative Medicine Use in the United States, $1990-1997$. JAMA. 1998;280:1569-75.

16. Graham RE, Gandhi TK, Borus J et al.

Risk of Concurrent Use of Prescription Drugs with Herbal and Dietary Supplements in Ambulatory Care. Advances in Patient Safety: From Research to Implementation. 2008;4:1-13.

17. Robinson A, McGrail MR. Disclosure of CAM use to medical practitioners: A review of qualitative and quantitative studies. Complement Ther Med. 2004;12:90-98. 
NOTICE: This is the author's version of a work accepted for publication by Elsevier. Changes resulting from the publishing process, including peer review, editing, corrections, structural formatting and other quality control mechanisms, may not be reflected in this document. Changes may have been made to this work since it was submitted for publication. The definitive version has been published in Complementary Therapies in Medicine, Volume 19, Issue 1, 2011. DOI: 10.1016/j.ctim.2010.12.005

18. Eisenberg DM, Kessler RC, Van Rompay MI, et al. Perceptions about complementary therapies relative to conventional therapies among adults who use both: Results from a national survey. Ann Intern Med. 2001;135:344352 .

19. Busse JW, Heaton G, Wu P, Wilson KR, Mills EJ. Disclosure of natural product use to primary care physicians: A cross-sectional survey of naturopathic clinic attendees. Mayo Clin Proc. 2005;80:6161-623.

20. Adler SR, Fosket JR. Disclosing complementary and alternative medicine use in the medical encounter: A qualitative study of women with breast cancer. J Fam Pract. 1999;48:453-458.

21. Pagan JA, Pauly MV. Access to conventional medical care and the use of complementary and alternative medicine. Health Aff. 2005;24:255-262.

22. Kroesen K, Baldwin CM, Brooks AJ, Bell IR. US military veterans' perceptions of the conventional medical care system and their use of complementary and alternative medicine. Fam Pract. 2002; 19: 57 - 64.

23. Astin JA. Why patients use alternative medicine: Results of a national study. JAMA. 1998;279: 1548-53.

24. Kessler RC, Davis RB, Foster DF, et al. Long-term trends in the use of complementary and alternative medical therapies in the United States. Ann Intern Med. 2001;135:262-268.

25. Swani A, Thomas R. Pediatricians' attitudes, experience and referral patterns regarding complementary/alternative medicine; a national survey. BMC Complement Altern Med. 2007;7(18). http://www.biomedcentral.com/1472-6882/7/18/. Accessed November 12, 2009.

26. Winslow LB, Shapiro H. Physicians want education about complementary and alternative medicine to enhance communication with their patients. Ann Intern Med. 2002;162:1176-1181.

27. Ambrose ET, Samuels S. Perception and use of herbals among students and their practitioners in a University setting. J Am Acad Nurse Pract. 2004;16:166-173.

28. Johnson SK, Blanchard A. Alternative medicine and herbal use among university students. J Am Coll Health. 2006;55:163-168.

29. Newberry H, Beerman K, Duncan S, McGuire M, Hillers V. Use of nonvitamin, nonmineral dietary supplements among college students. J Am Coll Health, 2001;50:123-129.

30. Radloff LS. The CES-D Scale: a self-report depression scale for research in the general population. App Psych Meas 1977; 1:385-401.

31. Westhuis D, Thyer BA. Development and validation of the Clinical Anxiety Scale: A rapid assessment instrument for empirical practice. Educ Psychol Meas. 1989;49:153-163. 
NOTICE: This is the author's version of a work accepted for publication by Elsevier. Changes resulting from the publishing process, including peer review, editing, corrections, structural formatting and other quality control mechanisms, may not be reflected in this document. Changes may have been made to this work since it was submitted for publication. The definitive version has been published in Complementary Therapies in Medicine, Volume 19, Issue 1, 2011. DOI: 10.1016/j.ctim.2010.12.005

Table 1. Key Differences in Participant Herb Users and Non Herb Users in the last 12 Months

\begin{tabular}{lcccccc}
\hline & \multicolumn{3}{c}{ Herb Users } & \multicolumn{2}{c}{ No Herb Use } & \multicolumn{2}{c}{ Total } \\
\cline { 2 - 7 } & $\mathrm{N}$ & $\%$ & $\mathrm{~N}$ & $\%$ & $\mathrm{~N}$ & $\%$ \\
\hline Gender & & & & & & \\
Male & 32 & 26.0 & 91 & 74.0 & 123 & 100 \\
$\quad$ Female & 57 & 34.1 & 110 & 65.9 & 167 & 100 \\
$\begin{array}{c}\text { Prescription Use } \\
\text { Yes }\end{array}$ & 59 & 34.9 & 110 & 65.1 & 169 & 100 \\
$\quad$ No & 30 & 24.0 & 95 & 76.0 & 125 & 100 \\
CES-D Score > 15 & & & & & & \\
Yes & 34 & 31.8 & 73 & 68.2 & 107 & 100 \\
$\quad$ No & 54 & 29.0 & 132 & 71.0 & 186 & 100 \\
CAS Score > 30 & & & & & & \\
Yes & 18 & 29.0 & 44 & 71.0 & 62 & 100 \\
No & 71 & 30.6 & 161 & 69.4 & 231 & 100 \\
\hline
\end{tabular}

Prescription use indicates use of prescription drugs in the past 12 months. A CES-D score of $>15$ indicates possible presence of depression and a CAS score of $>30$ may indicate anxiety. 
NOTICE: This is the author's version of a work accepted for publication by Elsevier. Changes resulting from the publishing process, including peer review, editing, corrections, structural formatting and other quality control mechanisms, may not be reflected in this document. Changes may have been made to this work since it was submitted for publication. The definitive version has been published in Complementary Therapies in Medicine, Volume 19, Issue 1, 2011. DOI: 10.1016/j.ctim.2010.12.005

Figure 1. Percentage of concurrent herb-drug use by herb used in the past 12 months.

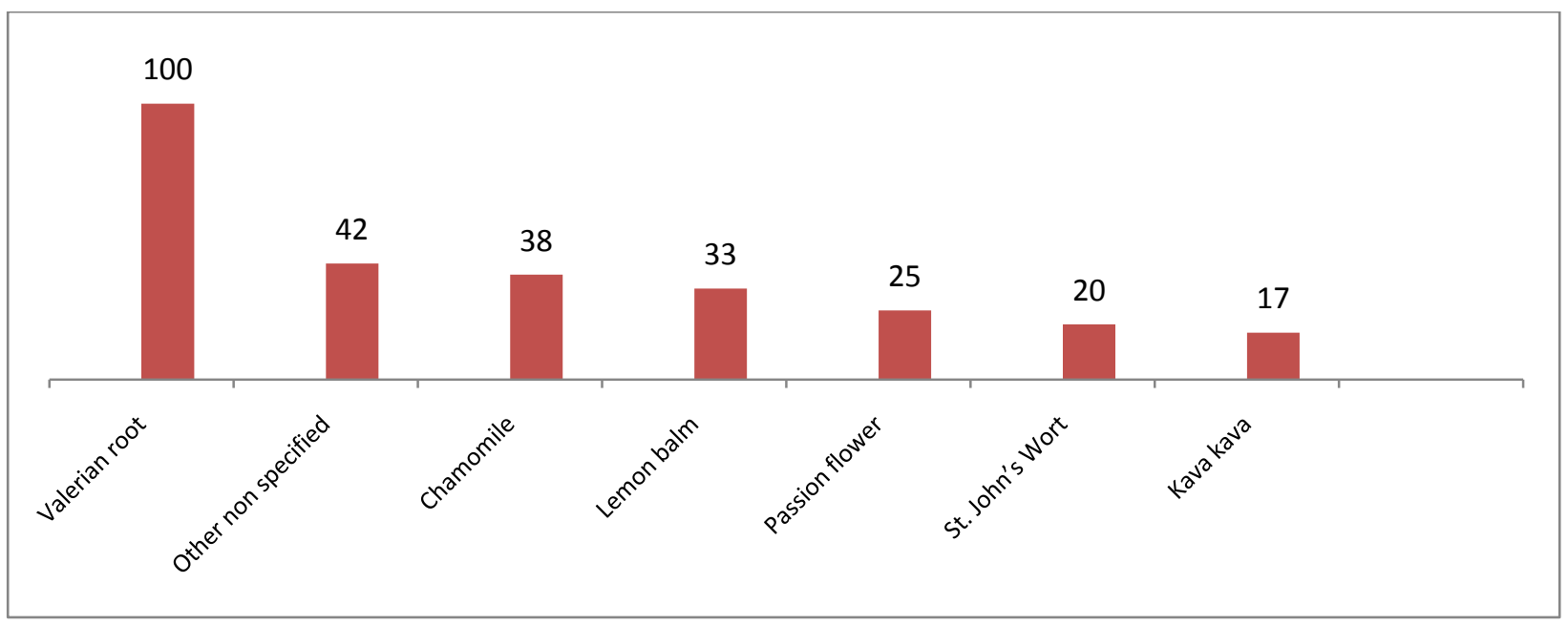


NOTICE: This is the author's version of a work accepted for publication by Elsevier. Changes resulting from the publishing process, including peer review, editing, corrections, structural formatting and other quality control mechanisms, may not be reflected in this document. Changes may have been made to this work since it was submitted for publication. The definitive version has been published in Complementary Therapies in Medicine, Volume 19, Issue 1, 2011. DOI: 10.1016/j.ctim.2010.12.005

Figure 2. Percent of herb use by drug type use in the past 12 months

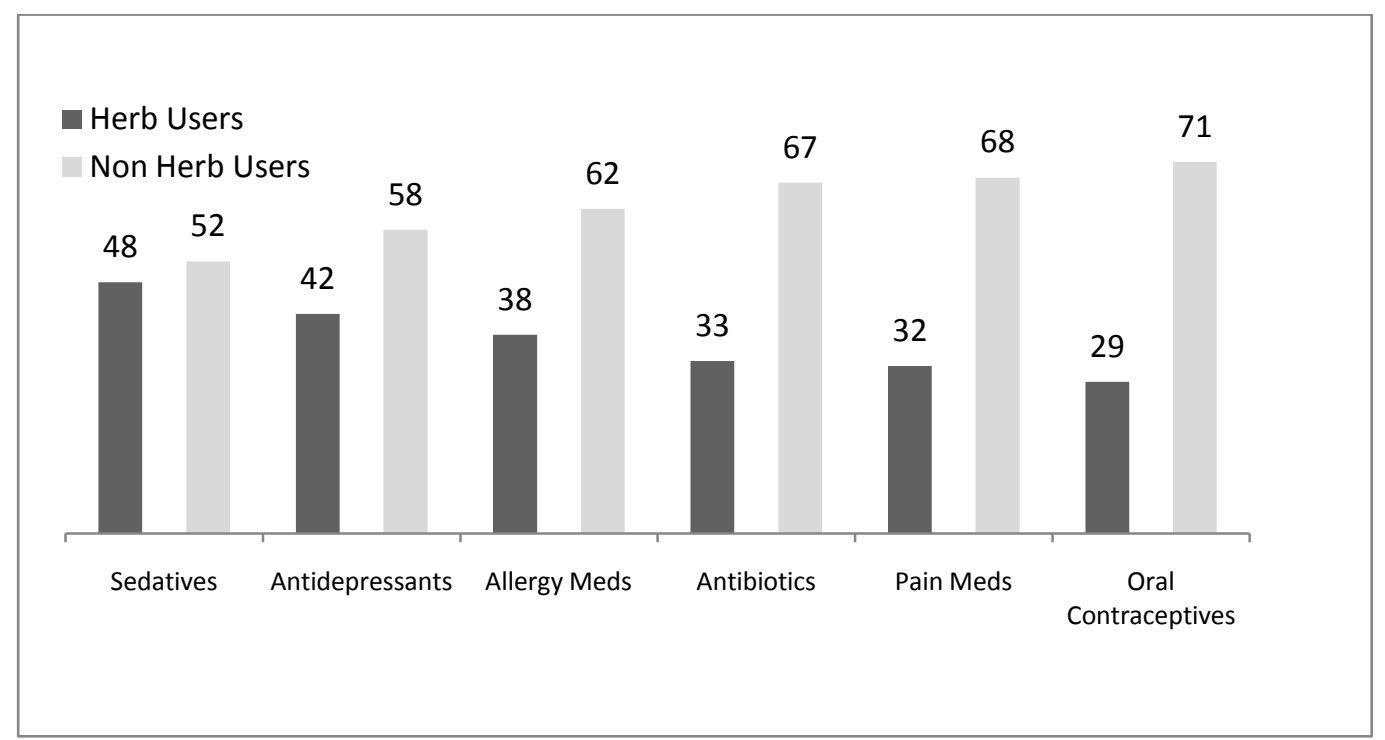

